\title{
АНАЛІТИЧНО-ЧИСЛОВЕ ВИЗНАЧЕННЯ СТАЦІОНАРНОГО ТЕПЛОВОГО СТАНУ ТЕРМОЧУТЛИВИХ БАГАТОШАРОВИХ СТРУКТУР ПРОСТОЇ ГЕОМЕТРIї
}

\author{
Ігор Махоркін ${ }^{1}$, к. ф.-М. н., Микола Махоркін ${ }^{1,3}$, к. ф.-М. н., \\ Тетяна Махоркіна ${ }^{2}$, к. ф.-м. н., Петро Пукач ${ }^{3}$, д. т. н. \\ ${ }^{1}$ Інститут прикладних проблем механіки і математики ім. Я. С. Підстригача НАН Украйни, \\ вул. Наукова 3-б, м. Львів, Украӥна, \\ e-mail:mahorkin@ukr.net \\ ${ }^{2}$ Львівський наиіональний аграрний університет, \\ вул. Володимира Великого, 1, м. Дубляни, Львівський р-н, Львівська обл., Украйна, \\ e-mail:mechanic@ua.fm \\ ${ }^{3}$ Наиіональний університет "Львівська політехніка", вул. С. Бандери, 12, м. Львів, Украӥна, \\ e-mail: petro.y.pukach@lpnu.ua
}

https://doi.org/10.31734/agroengineering2021.25.148

\begin{abstract}
Махоркін І., Махоркін М., Махоркіна Т., Пукач П. Аналітично-числове визначення стаціонарного теплового стану термочутливих багатошарових структур простої геометрії

Запропоновано та апробовано аналітично-числову методику визначення одномірного стаціонарного теплового стану багатошарових термочутливих структур простої геометрії незалежно від характеру температурних залежностей теплофізичних та механічних характеристик матеріалу шарів.

3 цією метою розглянуто багатошарові тіла 3 термочутливих матеріалів, віднесених до однієї з класичних ортогональних систем координат (декартової, циліндричної, сферичної), граничні поверхні та поверхні спряження матеріалів яких збігаються $з$ координатними поверхнями (багатошарові структури простої геометрії). Вважається, що тепловий стан, зумовлений термічним навантаженням, характеризується одновимірним стаціонарним температурним полем.

Грунтуючись на співвідношеннях нелінійної теорії теплопровідності неоднорідних тіл, сформульовано, у вигляді крайової задачі теплопровідності, математичну модель теплової поведінки таких структур. Ця модель полягає у визначенні температури як функції координати за розв'язками рівняння теплопровідності. При цьому їх теплофізичні й механічні характеристики як єдиного цілого подаються у вигляді кусково-постійних функцій координати та температури.

За допомогою введення у розгляд аналога функції Кірхгофа та використання апарату узагальнених функцій у замкнутому аналітичному вигляді побудовано аналітично-числові розв'язки нелінійних одновимірних стаціонарних задач теплопровідності шаруватих темочутливих тіл простої геометрії за довільного характеру температурної залежності фізико-механічних характеристик матеріалів шарів, що не потребують з'ясування їх однозначності.

На прикладі числового дослідження стаціонарного теплового стану та зумовленого ним статичного термопружного стану двошарової пластини, граничні поверхні якої перебувають в умовах конвективного теплообміну зі середовищами постійної температури, апробовано запропонований аналітично-числовий підхід та отримані на його основі аналітично-числові розв'язки.

Ключові слова: багатошарові структури, тіла простої геометрії, стаціонарний тепловий стан, температурозалежні фізико-механічні характеристики, узагальнені функції.
\end{abstract}

Makhorkin I., Makhorkin M., Makhorkina T., Pukach P. Analytical and numerical assessment of stationary thermal state of simple geometry thermosensitive multilayer structures

The analytical and numerical method to assess one-dimensional stationary thermal state of the multilayer thermosensitive structures of simple geometry, irrespective of the nature of temperature dependences of the thermophysical and mechanical characteristics of the layer material, is proposed and tested.

To achieve the set goal, multilayer bodies maded of thermosensitive materials, assigned to one of the classical orthogonal coordinate systems (Cartesian, cylindrical, spherical), whose boundary surfaces and conjugation surfaces of materials coincide with coordinate surfaces (multilayer structures of simple geometry) are considered. It is suggested that the thermal state due to thermal loading is characterized by a one-dimensional stationary temperature field.

Based on the relations of the theory of nonlinear thermal conductivity of inhomogeneous bodies, a mathematical model of thermal behavior of such structures is formulated in the form of a boundary value problem of thermal conductivity. This model consists in determining the temperature as a function of coordinate of solutions of the thermal conductivity equation. 
In this case, their thermophysical and mechanical characteristics are presented in the form of piecewise constant functions of coordinate and temperature.

The analytical- and numerical solutions of nonlinear one-dimensional stationary thermal conduction problem of layered thermosensitive bodies of simple geometry with arbitrary nature of temperature dependences of the physical and mechanical characteristics are constructed by introducing an analog of the Kirchhoff function and using the apparatus of generalized functions in a closed analytical form. These solutions do not require clarification of their uniqueness.

On the example of a numerical study of the steady thermal state and the predetermined static thermoelastic state of a twolayer plate, the limiting surfaces of which are in the conditions of convective heat exchange with constant temperature media, the proposed approach was tested and the analytical and numerical solutions were obtained.

Key words: multilayer structures, bodies of simple geometry, stationary thermal state, temperature-dependent physical and mechanical characteristics, generalized functions.

Постановка проблеми. Елементи конструкцій сучасного промислового обладнання, зокрема енергетичного, хімічного, кріогенного тощо, як правило, експлуатуються за умов інтенсивного термічного навантаження і $\epsilon$ здебільш неоднорідними функціонально-градієнтними (шаруватими) тілами простої геометрії (пластини, циліндри, кулі) або їх фрагментами. Очевидно, що на адекватність теоретичних досліджень теплової та термопружної поведінки таких елементів конструкцій суттєво впливає нехтування неоднорідністю, зокрема, функціональною градієнтністю (шаруватістю) елемента та залежністю від температури (термочутливістю) фізико-механічних характеристик (ФМХ) їхніх матеріалів. Крім того, значна частина методик прогнозування параметрів технологічних процесів виготовлення та експлуатації елементів конструкцій такого типу за високотемпературної дії грунтується на понятті ефективного джерела тепла [9], параметрами якого є теплофізичні характеристики матеріалів, характеристики технологічного процесу виготовлення чи експлуатації конструктивного елементу, його геометричної структури i, природно, термічної дії. У цих методиках приймається, що температурне поле є єдиною незалежною характеристикою, через яку визначаються всі інші. Дослідження згідно з цими методиками проводять у два етапи. На першому етапі формулюють i розв'язують теплову задачу, тобто визначають температурне поле, а на другому - температурне поле вважають відомим i розраховують швидкоплинні процеси та процеси, що не впливають на розподіл температури. Так, наприклад, дослідженню статичної чи квазістатичної термопружної поведінки тіл передує визначення їхнього теплового стану (температурного поля) [3; 10-13; 15-17].

Аналіз останніх досліджень і публікацій. Адекватне визначення теплового стану елементів конструкцій, у тому числі й шаруватих, за високотемпературної дії грунтується на моделі термочутливого тіла (модель враховує залежність фізико-механічних характеристик матеріалів від температури) [3; 13]. Відповідно до цієї моделі тепловий стан тіла визначається за розв'язком нелінійної задачі теплопровідності.

$\mathrm{У}$ роботах $[2 ; 4 ; 8]$ запропоновано методики визначення стаціонарних температурних полів у шаруватих тілах за лінійної, квадратичної та кубічної залежності коефіцієнтів теплопровідності матеріалів шарів від температури. Ці методики, за довільної кількості шарів, залежно від умов теплообміну за допомогою перетворення (заміни) Кірхгофа [3] зводять проблему до розв'язання одного або системи двох нелінійних алгебраїчних рівнянь, розв'язок яких рекомендується шукати числовими методами, зокрема методами послідовних наближень. При цьому вибір початкового наближення та 3'ясування існування і єдиності розв'язку потребує додаткових досліджень. 3 оглядом робіт, що стосуються окресленої тематики, можна ознайомитися в працях $[1-4 ; 6 ; 8 ; 10-$ $13 ; 15-17]$.

Постановка завдання. Наше завдання описати та апробувати запропонований авторами аналітично-числовий метод побудови розв'язків одновимірних стаціонарних задач теплопровідності багатошарових термочутливих тіл простої геометрії за високотемпературної дії. Цей метод грунтується на використанні апарату узагальнених функцій та уможливлює дослідження теплового стану шаруватих тіл незалежно від характеру температурних залежностей теплофізичних характеристик матеріалу шарів. Його використання також дає змогу уникнути з'ясування існування i єдиності розв'язку нелінійної задачі теплопровідності.

Постановка задачі. Розглянемо багатошарові тіла 3 термочутливих матеріалів, віднесені до однієї з класичних ортогональних систем координат $(\alpha, \beta, \gamma)$ (декартової- $x, y, z$ (рис. $1, a)$;

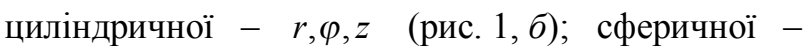


$r, \varphi, \theta \quad$ (рис. 1, в)). Граничні поверхні тіл збігаються 3 координатними поверхнями $(\alpha, \beta, \gamma)$ $\sim n_{i}=$ const $(i=0, n)$ (багатошарові структури простої геометрії [3]), а поверхні спряження матеріалів - iз координатними поверхнями $\alpha=\alpha_{i}=$ const $(i=\overline{1, n-1})$, на яких виконуються умови ідеального термоконтакту. Вважаємо, що тепловий стан, зумовлений термічним навантаженням, характеризується одновимірним стаціонарним температурним полем $t(\alpha)$.

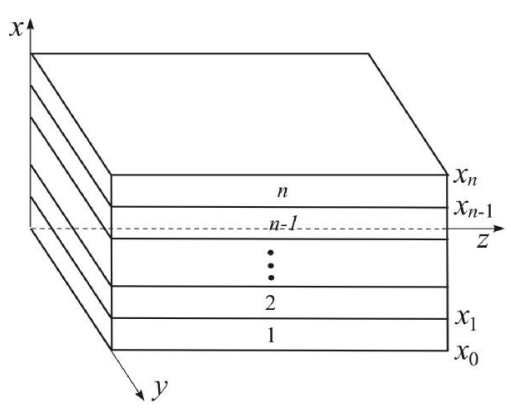

a)

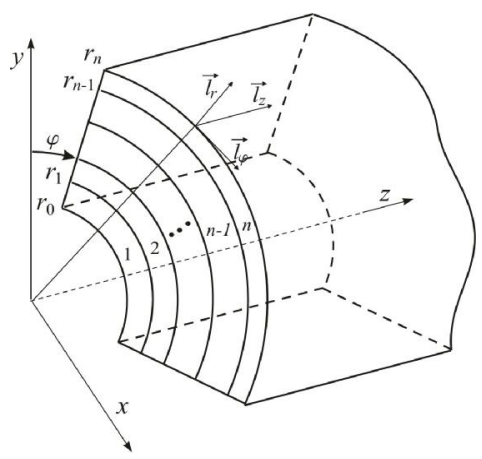

б)

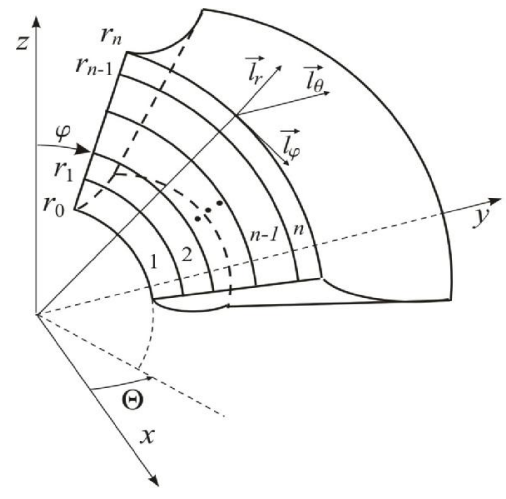

8)

Рис. 1. Багатошарові структури простої геометрії: $a$ - багатошарова пластина; $\sigma$ - багатошаровий циліндр; 8 - багатошарова куля

Fig. 1. Multilayer structures of simple geometry: $a$ - multilayer plate; $\sigma$ - multilayer cylinder; $b$ - multilayer sphere

Математичною моделлю теплової поведінки таких тіл, згідно з теорією неоднорідного тіла [5; 7], є нелінійна крайова задача стаціонарної теплопровідності, яка полягає у визначенні функції температури $t$ за розв'язком рівняння теплопровідності

$$
\left(\alpha^{k}\right)^{-1} \frac{\partial}{\partial \alpha}\left(\alpha^{k} \lambda_{t}(t, \alpha) \frac{\partial t}{\partial \alpha}\right)=-w_{t}(\alpha),
$$

що задовольняє умови ідеального термоконтакту на поверхнях спряження

$$
\left.t\right|_{\alpha_{i}-0}=\left.t\right|_{\alpha_{i}+0},\left.\left(\lambda_{t}(t, \alpha) \frac{d t}{d \alpha}\right)\right|_{\alpha_{i}-0}=\left.\left(\lambda_{t}(t, \alpha) \frac{d t}{d \alpha}\right)\right|_{\alpha_{i}+0},
$$

та граничні умови, які описують зовнішнє теплове навантаження

$$
\left.\left(a_{i}(t) \frac{d t}{d \alpha}+b_{i}(t)\right)\right|_{\alpha_{i}}=0,(i=0, n),
$$

де для: декартової системи координат $k=0, \alpha=x$; циліндричної - $k=1, \alpha=r$; сферичної $k=2, \alpha=r$, функції $a_{i}(t), b_{i}(t)$ вибираються відповідно до способу нагрівання, $w_{t}(\alpha)$ - густина потужності внутрішніх джерел-стоків тепла, усі похідні розуміються в класичному сенсі, а температурно-координатна залежність коефіцієнта теплопровідності $\lambda_{t}(t, \alpha)$ має вигляд $[1 ; 6]$ :

$$
\lambda_{t}(t, \alpha)=\lambda_{t}^{(1)}(t)+\sum_{i=1}^{n-1}\left(\lambda_{t}^{(i+1)}(t)-\lambda_{t}^{(i)}(t)\right) S_{+}\left(\alpha-\alpha_{i}\right),
$$

де $\lambda_{t}^{(i)}(t)$ - температурна залежність коефіцієнта теплопровідності матеріалу $i$-го шару.

Виклад основного матеріалу. Основні результати досліджень. В основу способу аналітично-числового розв'язування крайової задачі (1) - (3) покладено апроксимацію температурних залежностей коефіцієнтів теплопровідності матеріалу шарів $\lambda_{t}^{(i)}(t)$ кусково-постійними функціями температури виду [1]

$$
\begin{aligned}
\lambda_{t}^{(i)}(t) & =\Lambda^{(i)}(t) \approx \Lambda_{1}^{(i)}+\sum_{j=1}^{m}\left(\Lambda_{j+1}^{(i)}-\Lambda_{j}^{(i)}\right) S_{+}\left(t-t_{i}\right), \\
t_{0} & =t_{p}<t_{1}<t_{2}<\ldots<t_{m}<t_{k}=t_{m+1}
\end{aligned}
$$

та введення у розгляд функції типу функції Кірхгофа

$$
\vartheta(t)=\int_{0}^{t} \sum_{i=1}^{n} \Lambda^{(i)}(\xi) N_{i}(\alpha) d \xi,
$$

де $N_{i}(\xi)=S_{+}\left(\alpha-\alpha_{i-1}\right)-S_{+}\left(\alpha-\alpha_{i}\right)$, $\alpha_{0}<\alpha_{1}<\ldots<\alpha_{n-1}<\alpha_{n},\left[t_{p} ; t_{k}\right]-$ спільний температурний інтервал визначення $\lambda_{t}^{(i)}(t),(i=\overline{1, n})$, $\Lambda_{j}^{(i)}-$ із заданою точністю відповідає значенню $\lambda_{t}^{(i)}(t)$ в інтервалі температур $t_{j-1}<t<t_{j}, \alpha_{0}, \alpha_{n}-$ координати граничних поверхонь структури; $\alpha_{i}$ 
$(i=\overline{1, n-1})$ - координати поверхонь спряження (контакту), $i$-го та $i+1$-го шару, $S_{+}\left(\varsigma-\varsigma_{i}\right)=\left\{1, \varsigma>\varsigma_{i} ; 0, \varsigma \leq \varsigma_{i}\right\}$.

У результаті задача визначення стаціонарного теплового стану багатошарової структури зводиться до розв'язування співвідношення

$$
\begin{gathered}
\vartheta(t)=t \sum_{i=1}^{n} \Lambda^{(i)}(t) N_{i}(\alpha)- \\
-\sum_{i=1}^{n}\left[\sum_{j=1}^{m}\left(\Lambda_{j+1}^{(i)}-\Lambda_{j}^{(i)}\right) t_{j} S_{+}\left(t-t_{j}\right)\right] N_{i}(\alpha),
\end{gathered}
$$

за розв'язком частково виродженого рівняння

$$
\begin{array}{r}
\alpha^{-k} \frac{d}{d \alpha}\left\{\alpha^{k} \frac{d \vartheta}{d \alpha}-\sum_{i=1}^{n-1}\left(\left.\vartheta\right|_{\alpha_{i}+0}-\left.\vartheta\right|_{\alpha_{i}-0}\right) \alpha_{i}^{k} \delta_{+}\left(\alpha-\alpha_{i}\right)\right\}= \\
=\sum_{i=1}^{n}\left(\left.\frac{\tilde{d} \vartheta}{\tilde{d} \alpha}\right|_{\alpha_{i}+0}-\left.\frac{\tilde{d} \vartheta}{\tilde{d} \alpha}\right|_{\alpha_{i}-0}\right) \delta_{+}\left(\alpha-\alpha_{i}\right)-w_{t}(\alpha)
\end{array}
$$

отриманого $з$ рівняння теплопровідності (1) за допомогою апарату узагальнених функцій [7], $d / d \alpha$ - узагальнена похідна, $\tilde{d} / \tilde{d} \alpha$ - класична похідна.

Між $\vartheta$ та $t$ відповідно до співвідношення (6) існує взаємно однозначна відповідність, тому

$$
S_{+}\left(t-t_{i}\right)=S_{+}\left(\vartheta-\vartheta_{i}\right),
$$

$\mathrm{i}$, як наслідок, $\Lambda^{(i)}(t)=\Lambda^{(i)}(\vartheta)$, а

$$
\begin{gathered}
\vartheta(t)=t \sum_{i=1}^{n} \Lambda^{(i)}(\vartheta) N_{i}(\alpha)- \\
-\sum_{i=1}^{n}\left[\sum_{j=1}^{m}\left(\Lambda_{j+1}^{(i)}-\Lambda_{j}^{(i)}\right) t_{j} S_{+}\left(\vartheta-\vartheta_{j}\right)\right] N_{i}(\alpha) .
\end{gathered}
$$

Звідси отримуємо, що

$$
t=\left(\vartheta+\sum_{i=1}^{n} F_{i}(\vartheta) N_{i}(\alpha)\right)\left(\sum_{i=1}^{n} \Lambda^{(i)}(\vartheta) N_{i}(\alpha)\right)^{-1},
$$

де $F_{i}(\vartheta)=\sum_{j=1}^{m}\left(\Lambda_{j+1}^{(i)}-\Lambda_{j}^{(i)}\right) t_{j} S_{+}\left(\vartheta-\vartheta_{j}\right), \vartheta_{l}=\vartheta_{l}(\alpha)$,

$\vartheta_{l}(\alpha)=\sum_{i=1}^{n}\left[t_{l} \Lambda^{(i)}\left(t_{l}\right)-\sum_{j=1}^{m}\left(\Lambda_{j+1}^{(i)}-\Lambda_{j}^{(i)}\right) t_{j} S_{+}\left(t_{l}-t_{j}\right)\right] N_{i}(\alpha)$.

На поверхнях спряження $\alpha_{i}$ виконуються умови ідеального термоконтакту (2), тому, взявши до уваги (9), (10), можемо стверджувати справедливість співвідношень

$$
\begin{aligned}
& \left.t\right|_{\alpha_{i}-0}=\left.t\right|_{\alpha_{i}+0}=\left.t\right|_{\alpha_{i}},\left.\quad \vartheta\right|_{\alpha_{i}-0}=\left.\vartheta\right|_{\alpha_{i}}, \\
& \left.S_{+}\left(t-t_{i}\right)\right|_{\alpha_{i}}=\left.S_{+}\left(\vartheta-\vartheta_{i}\right)\right|_{\alpha_{i}}=\left.S_{+}\left(\vartheta-\vartheta_{i}\right)\right|_{\alpha_{i}+0}, \\
& \left.\vartheta\right|_{\alpha_{i}-0}=\left.\left(\Lambda^{(i)}(\vartheta) t-F_{i}(\vartheta)\right)\right|_{\alpha_{i}},\left.\quad \vartheta\right|_{\alpha_{i}+0}=\left.\left(\Lambda^{(i+1)}(\vartheta) t-F_{i+1}(\vartheta)\right)\right|_{\alpha_{i}}, \\
& \left.\left.\frac{\tilde{d} \vartheta}{\tilde{d} \alpha}\right|_{\alpha_{i}-0}=\left.\left(\Lambda^{(i)}(t) \frac{\tilde{d} t}{\tilde{d} \alpha}\right)\right|_{\alpha_{i}-0},\left.\quad \frac{\tilde{d} \vartheta}{\tilde{d} \alpha}\right|_{\alpha_{i}+0}=\left.\left(\Lambda^{(i)}(t) \frac{\tilde{d} t}{\tilde{d} \alpha}\right)\right|_{\alpha_{i}+0}, \quad\right\}
\end{aligned}
$$

спираючись на які отримуємо

$$
\begin{aligned}
& \left.\vartheta\right|_{\alpha_{i}+0}-\left.\vartheta\right|_{\alpha_{i}-0}=\left.K_{i} \vartheta\right|_{\alpha_{i}}+Q_{i},\left.\quad \frac{\tilde{d} \vartheta}{\tilde{d} \alpha}\right|_{\alpha_{i}+0}-\left.\frac{\tilde{d} \vartheta}{\tilde{d} \alpha}\right|_{\alpha_{i}-0}=0, \\
& K_{l}=\left.\left(\frac{\Lambda^{(l+1)}(\vartheta)}{\Lambda^{(l)}(\vartheta)}-1\right)\right|_{\alpha_{l}}, Q_{l}=\left.\left(\frac{\Lambda^{(l+1)}(\vartheta)}{\Lambda^{(l)}(\vartheta)} F_{l}-F_{l+1}(\vartheta)\right)\right|_{\alpha_{l}} .
\end{aligned}
$$

У результаті розв'язок рівняння (8) набуде такого вигляду:

$$
\vartheta=C_{1} f_{k}(\alpha)+C_{2}+\sum_{i=1}^{n}\left(\left.K_{i} \vartheta\right|_{\alpha_{i}}+Q_{i}\right) S_{+}\left(\alpha-\alpha_{i}\right)-W_{t} .
$$

Тут $f_{1}(\alpha)=\alpha-\alpha_{0}$ для пластини;

$$
f_{2}(\alpha)=\ln \frac{\alpha}{\alpha_{0}}-\text { циліндра; } f_{3}(\alpha)=\frac{\alpha_{i}-\alpha}{\alpha_{i} \alpha}-\text { кулі; }
$$$$
W_{t}(\alpha)=\int_{0}^{\alpha} \eta^{-k} \int_{0}^{\eta} \zeta^{k} w_{t}(\zeta) d \zeta d \eta \text {. }
$$

$$
\begin{aligned}
& \text { Подавши }\left.\quad \vartheta\right|_{\alpha_{i}} \quad \text { у } \quad \text { вигляді } \\
& \left.\vartheta\right|_{\alpha_{i}}=\tilde{K}_{1}^{(i)} C_{1}+\tilde{K}_{2}^{(i)} C_{2}+\tilde{K}_{3}^{(i)}, \quad 3 \quad \text { (16) отримано }
\end{aligned}
$$

рекурентні співвідношення для визначення $\tilde{K}_{j}^{(i)},(j=1,2,3)$ :

$$
\begin{gathered}
\tilde{K}_{1}^{(i)}=f_{k}\left(\alpha_{i}\right)+\sum_{j=1}^{i-1} K_{j} \tilde{K}_{1}^{(j)}, \tilde{K}_{2}^{(i)}=1+\sum_{j=1}^{i-1} K_{j} \tilde{K}_{2}^{(j)}, \\
\tilde{K}_{3}^{(i)}=\sum_{j=1}^{i-1}\left(K_{j} \tilde{K}_{3}^{(i)}+Q_{j}\right)-W\left(\alpha_{i}\right),
\end{gathered}
$$

у результаті чого вираз (16) для $\vartheta$ набуває вигляду

$$
\begin{aligned}
\vartheta=C_{1} & {\left[f_{k}(\alpha)+\sum_{j=1}^{n-1} K_{j} \tilde{K}_{1}^{(j)} S_{+}\left(\alpha-\alpha_{j}\right)\right]+C_{2}\left[1+\sum_{j=1}^{n-1} K_{j} \tilde{K}_{2}^{(j)} S_{+}\left(\alpha-\alpha_{j}\right)\right](18) } \\
& -\left[W_{t}(\alpha)-\sum_{j=1}^{n-1}\left(K_{j} \tilde{K}_{3}^{(i)}+Q_{j}\right) S_{+}\left(\alpha-\alpha_{j}\right)\right] .
\end{aligned}
$$

У загальному випадку значення констант інтегрування $C_{1}, C_{2}$ визначається із системи двох нелінійних алгебраїчних рівнянь, що отримуються за допомогою підстановки виразу (11) у граничні умови (3), які описують теплообмін структури 3 навколишнім середовищем (зовнішне теплове навантаження). Зауважимо, що в разі задання на одній з граничних поверхонь $\left(\alpha=\alpha_{i}, i=0, n\right)$ теплообміну I або II роду знаходження $C_{1}, C_{2}$ зводиться до розв'язання одного нелінійного алгебраїчного рівняння. У разі задання на одній 3 граничних поверхонь теплообміну I $\left(\left.t\right|_{\alpha_{0}}=t_{0, n}^{*}\right)$, a на іншій теплообміну II $\left(\left.\left(\lambda_{t}(t) \frac{\partial t}{\partial \alpha}\right)\right|_{\alpha_{0, n}}=q\right)$ роду отримаємо замкнутий аналітичний розв'язок.

Апробація запропонованого аналітичночислового підходу виконувалась на прикладі числового дослідження стаціонарного теплового 
стану та зумовленого ним статичного термопружного стану двошарової пластини (рис. 2), граничні поверхні $z_{0}=0, z_{2}=h_{2}$ якої вільні від силового навантаження та перебувають в умовах конвективного теплообміну зі середовищами постійної температури $t_{s}^{(0)}, t_{s}^{(n)}$ відповідно. Припускаємо також, що масові сили відсутні, а торцеві поверхні плити теплоізольовані та завантажені системою сил, головні вектор та момент якої дорівнюють нулю.

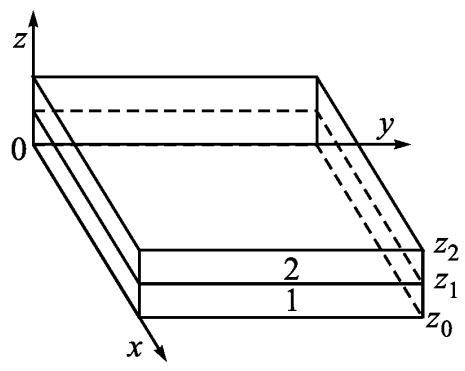

Рис. 2. Схема двошарової пластини

Fig. 2. Scheme of a two-layer plate

Температурне поле такої шаруватої структури згідно зі співвідношеннями (11), (15) - (18) визначається формулою

$$
t=\frac{\vartheta+F_{1}(\vartheta) S_{-}\left(\alpha_{1}-\alpha\right)+F_{2}(\vartheta) S_{+}\left(\alpha-\alpha_{1}\right)}{\Lambda^{(1)}(\vartheta) S_{-}\left(\alpha_{1}-\alpha\right)+\Lambda^{(2)}(\vartheta) S_{+}\left(\alpha-\alpha_{1}\right)},
$$

де

$$
\begin{aligned}
\vartheta & =C_{1}\left[f_{2}(\alpha)+K_{1} f_{2}\left(\alpha_{1}\right) S_{+}\left(\alpha-\alpha_{1}\right)\right]+ \\
& +C_{2}\left[1+K_{1} S_{+}\left(\alpha-\alpha_{1}\right)\right]+Q_{1} S_{+}\left(\alpha-\alpha_{1}\right),
\end{aligned}
$$

$$
K_{1}=\left.\left(\frac{\Lambda^{(2)}(\vartheta)}{\Lambda^{(1)}(\vartheta)}-1\right)\right|_{\alpha_{1}}, Q_{1}=\left.\left(\frac{\Lambda^{(2)}(\vartheta)}{\Lambda^{(1)}(\vartheta)} F_{1}-F_{2}(\vartheta)\right)\right|_{\alpha_{1}},
$$$$
S_{-}\left(\alpha_{1}-\alpha\right)=1-S_{+}\left(\alpha-\alpha_{1}\right)
$$

$$
F_{i}(\vartheta)=\sum_{j=1}^{m}\left(\Lambda_{j+1}^{(i)}-\Lambda_{j}^{(i)}\right) t_{j} S_{+}\left(\vartheta-\vartheta_{j}\right) \text {, }
$$

$$
\vartheta_{l}=\sum_{i=1}^{n}\left[t_{l} \Lambda^{(i)}\left(t_{l}\right)-\sum_{j=1}^{m}\left(\Lambda_{j+1}^{(i)}-\Lambda_{j}^{(i)}\right) t_{j} S_{+}\left(t_{l}-t_{j}\right)\right] N_{i}(\alpha) .
$$

Сталі інтегрування $\mathrm{C}_{1}, \mathrm{C}_{2}$ визначаються 3 граничних умов (3) за допомогою ітераційного циклу

$$
\left.\begin{array}{c}
C_{1}^{(\mathrm{n}+1)}=\varphi_{1}\left(\mathrm{C}_{1}^{(\mathrm{n})}, \mathrm{C}_{2}^{(\mathrm{n})}\right), \\
\mathrm{C}_{2}^{(\mathrm{n}+1)}=\varphi_{2}\left(\mathrm{C}_{1}^{(\mathrm{n})}, \mathrm{C}_{2}^{(\mathrm{n})},\right.
\end{array}\right\}
$$

Тут

$$
\left.\begin{array}{c}
\varphi_{1}\left(C_{1}, C_{2}\right)=\frac{\alpha_{2}^{*}\left[t_{s}^{(2)} \Lambda^{(2)}\left(\vartheta_{2}\right)-F_{2}\left(\vartheta_{2}\right)-Q_{1}-\left(1+K_{1}\right) C_{2}\right]}{z_{2} \alpha_{2}^{*}+\Lambda^{(2)}\left(\vartheta_{2}\right)+K_{1} \alpha_{2} z_{1}} \\
\varphi_{2}\left(\mathrm{C}_{1}, \mathrm{C}_{2}\right)=\Lambda^{(1)}\left(\vartheta_{0}\right)\left(\frac{C_{1}}{z_{0}}+t_{s}^{0}\right)+F_{1}\left(\vartheta_{0}\right),
\end{array}\right\}
$$

$$
\begin{gathered}
\vartheta_{0}=\vartheta\left(\mathrm{z}_{0}\right)=C_{2}, \\
\vartheta_{2}=\vartheta\left(z_{2}\right)=C_{1}\left[z_{2}+K_{1} h_{1}\right]+C_{2}\left[1+K_{1}\right]+Q_{1},
\end{gathered}
$$

За початкове наближення $\mathrm{C}_{1}, \mathrm{C}_{2}$ приймались їхні значення за постійного значення коефіцієнтів теплопровідності шарів

$$
\lambda_{t}^{(m)}(t)=\text { const }(m=\overline{1, n}) .
$$

Умовою припинення ітераційного процесу $\epsilon$ виконання співвідношення

$$
\max \left\{\frac{\left|t_{k+1}-t_{k}\right|}{t_{k+1}}, \frac{\left|C_{1}^{(k+1)}-C_{1}^{(k)}\right|}{C_{1}^{(k+1)}}, \frac{\left|C_{2}^{(k+1)}-C_{2}^{(k)}\right|}{C_{2}^{(k+1)}}\right\}<\varepsilon,
$$

де $\varepsilon$ - наперед задана точність обчислення.

Термонапружений стан плоскої багатошарової структури за прийнятого термосилового навантаження 3 точністю до принципу Сен-Венана описується співвідношеннями [3;8]:

$$
\sigma_{x x}=\sigma_{y y}=\sigma_{0}(z), \sigma_{z z}=\sigma_{x z}=\sigma_{y z}=\sigma_{x y}=0,
$$

де

$$
\sigma_{0}=\frac{E_{i}(t, z)}{1-v_{i}(t, z)}\left[A_{1}+z A_{2}-\Phi(t, z)\right] .
$$

Сталі інтегрування $A_{1}, A_{2}$ за умови, що головний вектор і головний момент напружень $\sigma_{x x}, \sigma_{y y}$ дорівнюють нулю, тобто

$$
\int_{0}^{z_{n}} \sigma_{x x} d z=\int_{0}^{z_{n}} z \sigma_{x x} d z=\int_{0}^{z_{n}} \sigma_{y y} d z=\int_{0}^{z_{n}} z \sigma_{y y} d z=0,
$$

визначаються за формулами

де

$$
A_{1}=\frac{b_{1} a_{22}-b_{2} a_{12}}{a_{11} a_{22}-a_{12}^{2}}, A_{2}=\frac{b_{2} a_{11}-b_{1} a_{12}}{a_{11} a_{22}-a_{12}^{2}},
$$

$$
\left.\begin{array}{l}
a_{11}=\sum_{i=1}^{n} \int_{z_{i-1}}^{z_{i}} \frac{E_{i}(t)}{1-v_{i}(t)} d z, a_{12}=\sum_{i=1}^{n} \int_{z_{i-1}}^{z_{i}} \frac{z E_{i}(t)}{1-v_{i}(t)} d z, a_{22}=\sum_{i=1}^{n} \int_{z_{i-1}}^{z_{i}} \frac{z^{2} E_{i}(t)}{1-v_{i}(t)} d z, \\
b_{1}=\sum_{i=1}^{n} \int_{z_{i-1}}^{z_{i}} \frac{E_{i}(t) \Phi_{i}(t)}{1-v_{i}(t)} d z, b_{2}=\sum_{i=1}^{n} \int_{z_{i-1}}^{z_{i}} \frac{z E_{i}(t) \Phi_{i}(t)}{1-v_{i}(t)} d z .
\end{array}\right\}
$$

Якщо скористатись апроксимацією температурних залежностей ФМХ матеріалу складових кусково-постійними функціями від температури виду (5) та врахувати при цьому взаємно однозначну відповідність між $\vartheta$ та $t$ (9), то температурно-координатну залежність ФМХ пакету, як єдиного цілого, можемо подати у вигляді

$$
\begin{gathered}
\left\{\lambda_{t}, E, v, \alpha_{t}\right\} \sim p(t, z) \approx \tilde{p}(\vartheta, z)= \\
=\sum_{i=1}^{n}\left[\tilde{p}_{i 1}+\sum_{j=1}^{m}\left(\tilde{p}_{i j+1}-\tilde{p}_{i j}\right) S_{+}\left(\vartheta-\vartheta_{i j}\right)\right] N_{i}(z), \\
\vartheta_{i j}=\int_{0}^{t_{j}} \lambda_{t i}(\zeta) d \xi \approx \sum_{i=1}^{n}\left[t_{j} \tilde{\lambda}_{t i}\left(t_{j}\right)-\right. \\
\left.-\sum_{l=1}^{m-1}\left(\tilde{\lambda}_{t i}^{(l+1)}-\tilde{\lambda}_{t i}^{(l)}\right) t_{j} S_{+}\left(t_{j}-t_{l}\right)\right] N_{i}(z),
\end{gathered}
$$


$t_{0}=t_{p}<t_{1}<t_{2}<\ldots<t_{m}<t_{k}=t_{m+1}-\quad$ спільні вузли апроксимації, $\left[t_{p} ; t_{k}\right]$ - інтервал визначення ФМХ, $\tilde{p}_{i j}$ - значення коефіцієнтів апроксимації температурної залежності $p_{i}(t)$, відповідної ФМХ $i$-го шару, які із заданою точністю відповідають їх значенню в інтервалах температур $t_{j-1}<t<t_{j}$.

У такому разі їх алгебричні комбінації згідно 3 [7] мають такий самий вигляд (24) i співвідношення (21), (23), подаються в такому вигляді:

$$
\begin{aligned}
& \sigma_{0}=\tilde{L}(\vartheta, z)\left[A_{1}+z A_{2}-\tilde{\Phi}(\vartheta, z)\right], \\
& a_{11}=\int_{0}^{z_{n}} \tilde{L}(\vartheta, z) d z, a_{12}=\int_{0}^{z_{n}} z \tilde{L}(\vartheta, z) d z, a_{22}=\int_{0}^{z_{n}} z^{2} \tilde{L}(\vartheta, z) d z \\
& b_{1}=\int_{0}^{z_{n}} \tilde{L}(\vartheta, z) \tilde{\Phi}(\vartheta, z) d z, b_{2}=\int_{0}^{z_{n}} z \tilde{L}(\vartheta, z) \tilde{\Phi}(\vartheta, z) d z . \\
& \text { Тут } \quad \tilde{L}(\vartheta, z)=\sum_{i=1}^{n}\left[\frac{\tilde{E}_{i 1}}{1-\tilde{v}_{i 1}}+\right. \\
& \left.+\sum_{j=1}^{m}\left(\frac{\tilde{E}_{i j+1}}{1-\tilde{v}_{i j+1}}-\frac{\tilde{E}_{i j}}{1-\tilde{v}_{i j}}\right) S_{+}\left(\vartheta-\vartheta_{i j}\right)\right] N_{i}(z), \\
& \tilde{\Phi}(\vartheta, z)=\sum_{i=1}^{n}\left[\frac{\tilde{\alpha}_{t i}^{(1)}}{\tilde{\lambda}_{t i}^{(1)}} \vartheta+\right. \\
& \left.+\sum_{j=1}^{m}\left(\frac{\tilde{\alpha}_{t i}^{(j 1)}}{\tilde{\lambda}_{t i}^{(j+1)}}-\frac{\tilde{\alpha}_{t i}^{(j)}}{\tilde{\lambda}_{t i}^{(j)}}\right)\left(\vartheta-\vartheta_{i j}\right) S_{+}\left(\vartheta-\vartheta_{i j}\right)\right] N_{i}(z) \text {. }
\end{aligned}
$$

Співвідношення (25), (26) дають змогу досліджувати термопружну поведінку шаруватого плоского пакету безпосередньо за розв'язком відповідної крайової задачі для рівняння (8) на визначення функції типу функції Кірхгофа (6) та уникнути при цьому необхідності 3'ясування єдиності розв'язку відповідної нелінійної задачі теплопровідності.

Якщо прийняти, що ФМХ матеріалів шарів однакові, то співвідношення (19), (25), (26) будуть описувати тепловий та зумовлений термопружний стани однорідної плити.

Результати числових досліджень. Числові дослідження виконувались для гіпотетичної плити, виготовленої з Aluminum oxide (технічної кераміки) та сплаву Ti-6Al-4V. Температурні залежності ФМХ в інтервалі температур $\left[t_{p}=273 K ; t_{k}=873 K\right]$ їх визначення приймались у такому вигляді:
- для Aluminum oxide (технічної кераміки) $[3 ; 14]$ :

$\lambda_{t}(t)=\left(1,5828 \cdot 10^{4} t^{-1}-14,087+8,772 \cdot 10^{-2} t\right)\left[\frac{B m}{M \cdot K}\right]$,

$E(t)=\left(3,4955 \cdot 10^{11}-1,3468 \cdot 10^{8} t+1,4076 \cdot 10^{5} t^{2}\right)[\Pi a]$,

$\alpha_{t}(t)=\left(6,8269 \cdot 10^{-6}+1,2548 \cdot 10^{-9} t\right)\left[K^{-1}\right]$,

$v(t)=236,283 \cdot 10^{-3}-31,7412 \cdot 10^{-6} t+71,2602 \cdot 10^{-9} t^{2}$;

- для сплаву Ti-6Al-4V [14]:

$\lambda_{t}(t)=\left(1+1,704 \cdot 10^{-2} t\right)\left[\frac{B m}{M \cdot K}\right]$,

$E(t)=\left(122,56 \cdot 10^{9}-56,206 \cdot 10^{9} t\right)[\Pi a]$,

$\alpha_{t}(t)=\left(7,5788 \cdot 10^{-6}+5,03081 \cdot 10^{-9} t-23,8505 \cdot 10^{-12} t^{2}\right)\left[K^{-1}\right]$,

$v(t)=288,4 \cdot 10^{-3}+32,3296 \cdot 10^{-6} t$.

Під час досліджень відповідні температурні залежності ФМХ матеріалу шару $\left\{\lambda_{t}, E, v, \alpha_{t}\right\} \sim p(t)$ на температурному інтервалі їх визначення $t \in\left[t_{p} ; t_{k}\right]$ апроксимувались виразом (5), в якому коефіцієнти апроксимації $p_{j}^{(i)}$ та вузли апроксимації $t_{j}$ задавались таким чином:

$$
p_{j}^{(i)}=p^{(i)}\left(t_{j}\right), \quad t_{j}=t_{p}+j\left(t_{k}-t_{p}\right) m^{-1}, j=\overline{1, m},
$$

де $m$ - кількість вузлів апроксимації.

Характерні результати числових досліджень у графічному вигляді подано на рис. 3-5.

Графіки температурозалежності коефіцієнта теплопровідності $\lambda_{t}(t)$ технічної кераміки (суцільна крива), його кусково-постійної апроксимації $\Lambda(t)$ та відносної похибки $\varepsilon(t)=\frac{|\lambda(t)-\Lambda(t)|}{\lambda(t)} 100 \%$ такої апроксимації (штрихова крива) наведені на рис. 3, $a, \sigma$ у випадку 12 вузлів апроксимації (див. рис. $3, a$ ) та 24 вузлів (див. рис. 3 , б).

Розподіл значень температури $t(\tilde{z})$ (рис. 4, a) та напружень $\sigma(\tilde{z})$ (рис. 4, б) по товщині $\tilde{z}=z / h_{2}$, розрахованих за дійсних температурних залежностей ФМХ (суцільна крива) та різної кількості вузлів їх апроксимації $(m=3,6,12$ (відповідно криві $1,2,3)$ в однорідній плиті зі сплаву Ti-6Al-4Vпри $B i_{0}=\frac{\alpha_{0} h_{2}}{\lambda_{1}^{*}}=292, t_{s}^{(0)}=224 K$ та $\left.B i_{2}=\frac{\alpha_{2} h_{2}}{\lambda_{2}^{*}}=13, t_{s}^{(2)}=970 K\right)$ подано на рис. 4 . 


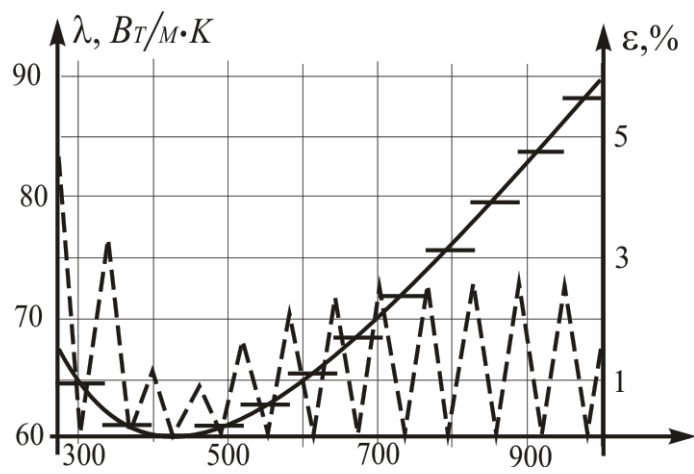

a)

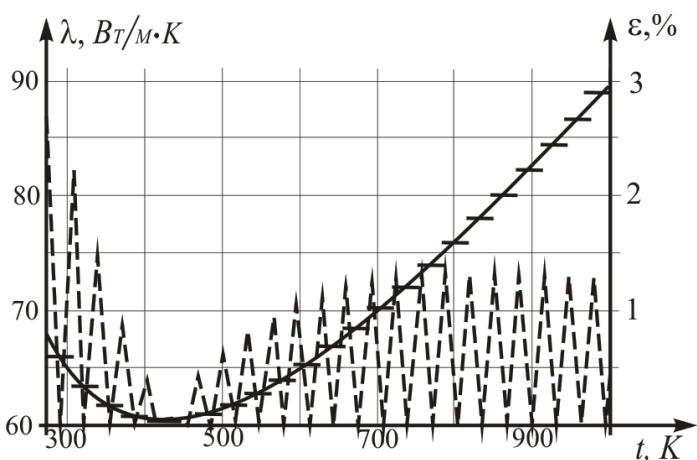

б)

Рис. 3. Залежність коефіцієнта теплопровідності $\lambda_{t}(t)$ технічної кераміки від температури.

Точне значення (неперервна крива), його кусково-постійна апроксимація та відносна похибка такої апроксимації (штрихова крива) при 12 вузлах апроксимації (a) та 24 вузлах (б)

Fig. 3. Dependence of heat conductivity $\lambda_{t}(t)$ of technical ceramics on temperature.

The exact value (continuous curve), its piecewise constant approximation and the relative error of such an approximation (dashed curve) at 12 nodes of approximation (a) and 24 nodes (б)

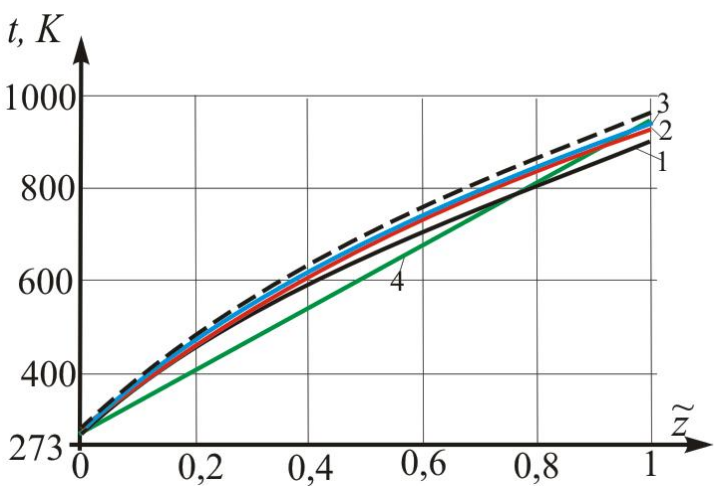

a)

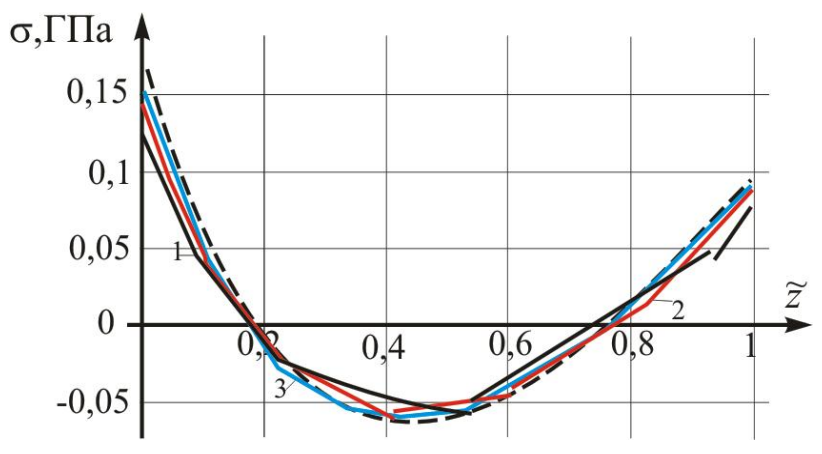

б)

Рис. 4. Розподіл значень температури $t(\tilde{z})(a)$ та напружень $\sigma(\tilde{z})$ (б) по товщині $\tilde{z}=z / h_{2}$, розрахованих за дійсних температурних залежностей ФМХ (штрихова крива) та різної кількості вузлів їх апроксимації $(m=3,6,12$ (відповідно криві 1, 2, 3) в однорідній плиті зі сплаву Ti-6Al-4V

$$
\text { при } \left.B i_{0}=\frac{\alpha_{0} h_{2}}{\lambda_{1}^{*}}=292, t_{s}^{(0)}=224 K \text { та } B i_{2}=\frac{\alpha_{2} h_{2}}{\lambda_{2}^{*}}=13, t_{s}^{(2)}=970 K\right)
$$

Fig. 4. Distribution of temperature $t(\tilde{z})$ values $(a)$ and stresses $\sigma(\tilde{z})(\sigma)$ by thickness $\tilde{z}=z / h_{2}$ calculated according to the actual temperature dependences of physical and mechanical characteristics (dashed curve) and different number of the approximation nodes $(m=3,6,12$, respectively, curves $1,2,3)$ in a homogeneous plate of Ti-6Al-4V alloy

$$
\text { at } \left.B i_{0}=\frac{\alpha_{0} h_{2}}{\lambda_{1}^{*}}=292, t_{s}^{(0)}=224 K \text { and } B i_{2}=\frac{\alpha_{2} h_{2}}{\lambda_{2}^{*}}=13, t_{s}^{(2)}=970 K\right)
$$

Розподіл значень температури $t(\tilde{z})$ (рис. 5, $a$ ) і значень напружень $\sigma(\tilde{z})$ (рис. $5, \sigma, 6)$ по товщині $\tilde{z}=z / h_{2}$, розрахованих за: дійсних температурних залежностей ФМХ (штрихова крива); різної кількості вузлів їх апроксимації $(m=3,6,12$, відповідно криві 1, 2, 3); апроксимації всіх ФМХ їх середньоінтегральними значеннями (крива 4); лише коефіцієнта теплопровідності (крива 5) у двошаровій плиті з технічної кераміки та сплаву Ті6Al-4V при $h_{1} / h_{2}=0,8, \quad B i_{0}=\frac{\alpha_{0} h_{2}}{\lambda_{1}^{*}}=44$, $t_{s}^{(0)}=221 K \quad$ та $\quad B i_{2}=\frac{\alpha_{2} h_{2}}{\lambda_{2}^{*}}=13, t_{s}^{(2)}=1050 K$, наведено на рис. 5. 


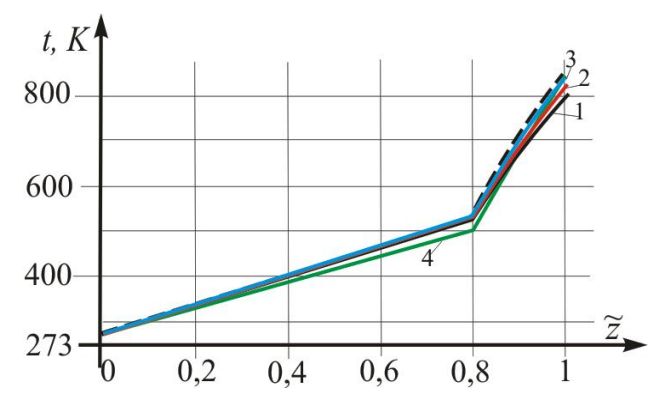

a)

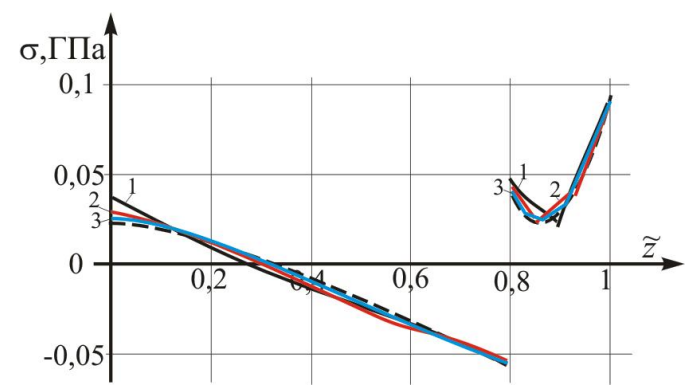

б)

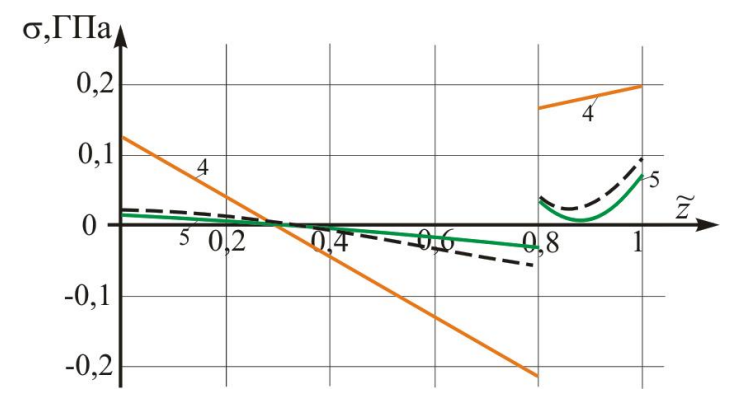

в)

Рис. 5. Розподіл значень температури $t(\tilde{z})(a)$ і значень напружень $\sigma(\tilde{z})(\sigma, 8)$ по товщині $\tilde{z}=z / h_{2}$, розрахованих за: дійсних температурних залежностей ФМХ (штрихова крива); різної кількості вузлів їх апроксимації ( $m=3,6,12$, відповідно криві $1,2,3)$; апроксимації всіх ФМХ їх середньоінтегральними значеннями (крива 4), лише коефіцієнта теплопровідності (крива 5) у двошаровій плиті з технічної кераміки

та сплаву Ti-6Al-4V при $h_{1} / h_{2}=0,8, B i_{0}=\frac{\alpha_{0} h_{2}}{\lambda_{1}^{*}}=44, t_{s}^{(0)}=221 K$ та $B i_{2}=\frac{\alpha_{2} h_{2}}{\lambda_{2}^{*}}=13, t_{s}^{(2)}=1050 \mathrm{~K}$

Fig. 5. Distribution by thickness $\tilde{z}=z / h_{2}$ of temperature values $t(\tilde{z})(a)$ and stress values $\sigma(\tilde{z})(\sigma, b)$ calculated by: actual temperature dependences of physical and mechanical characteristics (dashed curve): different number of nodes of their approximation $(m=3,6,12$, respectively, curves $1,2,3)$; approximations of all physical and mechanical characteristics by their average integral values (curve 4), only the heat conductivity coefficient (curve 5) in a two-layer plate of technical ceramics and Ti-6Al-4V alloy

$$
\text { at } h_{1} / h_{2}=0,8, B i_{0}=\frac{\alpha_{0} h_{2}}{\lambda_{1}^{*}}=44, t_{s}^{(0)}=221 \mathrm{~K} \text { and } B i_{2}=\frac{\alpha_{2} h_{2}}{\lambda_{2}^{*}}=13, t_{s}^{(2)}=1050 \mathrm{~K}
$$

Таблиця. Залежність максимального значення приведеної відносної похибки $\varepsilon_{F}=\left|\frac{F-F^{*}}{\widetilde{\Delta F}}\right|$ від кількості вузлів апроксимації

Table. Dependence on the number of approximation nodes of the maximum of the reduced relative error value $\varepsilon_{F}=\left|\frac{F-F^{*}}{\widetilde{\Delta F}}\right|$

\begin{tabular}{|c|c|c|c|c|c|}
\hline \multirow{2}{*}{\multicolumn{2}{|c|}{ Кількість вузлів апроксимації }} & \multicolumn{2}{|c|}{ Однорідна плита } & \multicolumn{2}{|c|}{ Двошарова плита } \\
\hline & & \multirow{2}{*}{$\begin{array}{c}\varepsilon_{t} \% \\
\overline{\Delta t}=800 \mathrm{~K} \\
7,87\end{array}$} & \multirow{2}{*}{$\begin{array}{c}\varepsilon_{\sigma} \% \\
\overline{\Delta \sigma}=2,35 \cdot 10^{8} \Pi a\end{array}$} & \multirow{2}{*}{$\begin{array}{c}\begin{array}{c}\varepsilon_{t} \% \\
\overline{\Delta t}\end{array}=800 \mathrm{~K} \\
3,84\end{array}$} & \multirow{2}{*}{$\begin{array}{c}\varepsilon_{\sigma} \% \\
\overline{\Delta \sigma}=1,53 \cdot 10^{8} \Pi a \\
10,68\end{array}$} \\
\hline & 3 & & & & \\
\hline & 6 & 4,6 & 11,05 & 2,11 & 5,25 \\
\hline & 12 & 2,54 & 6,61 & 1,01 & 2,84 \\
\hline & 24 & 1,33 & 3,54 & 0,46 & 1,5 \\
\hline \multirow{2}{*}{$\begin{array}{c}\text { За серед- } \\
\text { ньоінте- } \\
\text { гральних } \\
\text { значень } \\
\text { ФМХ }\end{array}$} & У cix ФМX & 11,88 & $\approx 70$ & 4,65 & 103,6 \\
\hline & $\begin{array}{c}\text { Лише коефіцієнт } \\
\text { теплопровідності } \\
\lambda_{t}(t)\end{array}$ & 11,88 & 36,6 & 4,65 & 18,34 \\
\hline
\end{tabular}


У таблиці подано результати, що ілюструють поведінку максимального значення приведеної відносної похибки $\varepsilon_{F}=\left|\frac{F-F^{*}}{\widetilde{\Delta F}}\right|$ залежно від кількості вузлів апроксимації $\left(F, F^{*}-\right.$ відповідно точне та наближене значення функції; $\left.\widetilde{\Delta F}=F_{\max }-F_{\min }\right)$.

Висновки. Наведені результати засвідчують, що:

- запропонована процедура забезпечує швидку збіжність процесу числового визначення теплового та зумовленого ним термонапруженого стану - збільшення числа вузлів апроксимації удвічі призводило в досліджуваних випадках до зменшення максимального значення приведеної відносної похибки приблизно у два рази;

- неврахування характеру температурозалежності ФМХ матеріалів шарів зазвичай призводить до значних похибок в оцінці теплового та термопружного стану системи як у кількісному, так і в якісному сенсі;

- формальне використання під час попередніх розрахунків апроксимації температурних залежностей ФМХ постійними величинами, рівними їх середньоінтегральним значенням, найімовірніше призведе до неадекватної оцінки термопружного стану об'єкта.

Запропонований аналітично-числовий підхід до розв'язання одновимірних задач стаціонарної теплопровідності та статичних задач термопружності плоских шаруватих структур із термочутливих матеріалів дає змогу досліджувати тепловий та напружений стани для різного характеру температурних залежностей ФМХ їхніх складових.

\section{Бібліографічний список}

1. Кушнір Р. М., Махоркін I. М., Махоркін М. I. Аналітично-числове визначення статичного термопружного стану плоских багатошарових термочутливих структур. Мат. методи $i$ фіз.-мех. поля. 2019. № 4. C. $131-140$.

2. Кушнір Р. М., Попович В. С. Про визначення усталеного термопружного стану багатошарових структур за високотемпературного нагрівання. Вісник Київського національного університету ім. Тараса Шевченка. Сер. Фіз.-мат. науки. 2013. № 3. С. 42-47.
3. Кушнір Р. М., Попович В. С. Термопружність термочутливих тіл. Моделювання та оптимізація в термомеханіці електропровідних тіл. Львів: СПОЛОМ, 2009. 412 c.

4. Кушнір Р. М., Процюк Ю. Б. Термопружний стан шаруватих тіл обертання за квадратичної залежності коефіцієнта теплопровідності. Фіз.-хім. механіка матеріалів. 2010. № 1. С. 7-18.

5. Ломакін В. А. Теория упругости неоднородных тел. Москва: Изд-во МГУ, 1976. 376 с.

6. Махоркін І.М., Махоркін М.І., Мастикаш Л. В. Аналітично-числове визначення термопружного стану багатошарових транстропних тіл простої геометрії. Прикл. пробл. мех. і мат. 2016. Т. 14. С. 133-139.

7. Подстригач Я.С., Ломакин В.А., Коляно Ю.М. Термоупругость тел неоднородной структуры. Москва: Наука, 1984. 368 с.

8. Процюк Ю. Б. Статичні задачі термопружності для шаруватих термочутливих плит за кубічної залежності коефіцієнта теплопровідності від температури. Мат. методи та фіз.-мех. поля. 2010. № 4. C. $151-162$.

9. Рыкалин Н. Н. Предисловие. Воздействие концентрированных потоков энергии на материалы. Москва: Наука, 1985. 246 с.

10. Carpinteri A., Paggi M. Thermo-elastic mismatch in nonhomogeneous beams. J. Eng. Math. 2008. No. 2-4. P. 371-384.

11. Noda N. Thermal stresses in materials with temperature-dependent properties. Appl. Mech. Rev. 1991. No. 44. P. 383-397.

12. Ootao Y., Tanigawa O., Ishimaru O. Optimization of material composition of functionality graded plate for thermal stress relaxation using a genetic algorithm. J. Therm. Stresses. 2000. No. 23. P. 257-271.

13. Popovych V. Methods for Determination of the Thermo-stressed State of Thermosensitive Solids Under Complex Heat Exchange Conditions. Encyclopedia of Thermal Stresses. Springer. 2014. No. 6. P. 2997-3008.

14. Shen, Hui-Shen. Functionally graded materials: nonlinear analysis of plates and shells. CRC Press, 2009. $280 \mathrm{p}$.

15. Tanigawa Y., Akai T., Kawamura R. Transient heat conduction and thermal stress problems of a nonhomogeneous plate with temperature-dependent material properties. J. Therm. Stresses. 1996. No. 1. P. 77-102.

16. Tanigawa Y., Ootao Y. Transient thermoelastic analysis of functionally graded plate with temperaturedependent material properties taking into account the thermal radiation. Nihon Kikai Gakkai Nenji Taikai Koen Ronbunshu. 2002. No. 2. P. 133-134.

17. Yangiian $\mathrm{Xu}$, Daihui Tu. Analysis of steady thermal stress in a $\mathrm{ZrO} 2 / \mathrm{FGM} / \mathrm{Ti}-6 \mathrm{Al}-4 \mathrm{~V}$ composite ECBF plate with temperature-dependent material properties by NFEM. WASE Int. Conf. on Inform. Eng. 2009. Vol. 02. P. 433-436. 